Pacific Journal of 


\title{
WEAKLY HYPERCENTRAL SUBGROUPS OF FINITE GROUPS
}

\author{
Donald C. Dykes
}

In this article the study of generalized Frattini subgroups of finite groups, developed by J. C. Beidleman and T. K. Seo, is continued. We call a proper normal subgroup $H$ of a finite group $G$, a special generalized Frattini subgroup of $G$ provided that $G=N_{G}(A)$ for each normal subgroup $L$ of $G$ and each Hall subgroup $A$ of $L$ such that $G=H N_{G}(A)$. Z. Janko proved that a subnormal subgroup $K$ of a finite group $G$ is $\pi$-closed, $\pi$ is a set of primes, whenever $K /(K \cap \phi(G))$ is $\pi$-closed, where $\phi(G)$ denotes the Frattini subgroup of $G$. We prove that a subnormal subgroup $K$ of a finite group $G$ is $\pi$-closed whenever $K /(K \cap H)$ is $\pi$-closed where $H$ is a special generalized Frattini subgroup of $G$. From this result we prove that a proper normal subgroup $H$ of a finite group $G$ is a special generalized Frattini subgroup of $G$ if and only if $H$ is a weakly hypercentral subgroup of $G$.

The properties of weakly hypercentral subgroups were developed by $R$. Baer. We obtain some of Baer's results in a different manner by using special generalized Frattini subgroups, and we also extend some of the properties of $\phi(G)$ to the class of generalized Frattini subgroups.

Some examples of special generalized Frattini subgroups of a finite group $G$ are the Frattini subgroup $\phi(G)$, the center $Z(G)$ of a nonabelian group $G$, and the intersection $L(G)$ of all self-normalizing maximal subgroups of a nonnilpotent group $G$.

\section{Notation.}

$G$ will always denote a finite group.

$|G|$ denotes the order of $G$.

$|G: H|$ is the index of the subgroup $H$ in $G$.

$H^{x}=x^{-1} H x$ where $x \in G$ and $H \leqq G$.

$Z(G)$ is the center of $G$.

$Z^{*}(G)$ is the hypercenter of $G$ (i.e., the terminal member of the upper central series of $G$ ).

$G^{\prime}$ denotes the commutator subgroup of $G$.

$D(G)$ denotes the hypercommutator of $G$ (i.e., the terminal member of the lower central series of $G$ ).

$N_{G}(H)$ denotes the normalizer of $H$ in $G$.

$\phi(G)$ is the Frattini subgroup of $G$. 
If $\pi$ is a set of primes, then $\pi^{\prime}$ is the set of primes not in $\pi$.

If $n$ is a positive integer and if $\pi$ is a set of primes, then $n$ is a $\pi$-number provided that each prime divisor of $n$ is in $\pi$.

The element $x$ of $G$ is a $\pi$-element if and only if $|x|$ is a $\pi$-number.

$G$ is a $\pi$-group provided that each of its elements is a $\pi$-element. of $G$.

$G$ is $\pi$-closed if the totality of $\pi$-elements in $G$ forms a subgroup

If $H \leqq G$, then $H$ is a Hall $\pi$-subgroup of $G\left(H \in \operatorname{Hall}_{\pi}(G)\right)$ if $H$ is a $\pi$-group and if $|H|$ and $|G: H|$ are relatively prime.

If $H \leqq G$, then $H$ is a Hall subgroup of $G$ provided that $|H|$ and $|G: H|$ are relatively prime.

$L(G)$ is the intersection of all self-normalizing maximal subgroups of $G$; set $L(G)=G$ if $G$ is nilpotent.

3. Special generalized Frattini subgroups. The concept of a generalized Frattini subgroup was developed in [3]. Recall that a proper normal subgroup $H$ of a group $G$ is called a generalized Frattini subgroup of $G$ if and only if $G=N_{G}(P)$ for each normal subgroup $L$ of $G$ and each Sylow $p$-subgroup $P$ of $L, p$ is a prime, such that $G=H N_{G}(P)$. We denote the collection of all generalized Frattini subgroups of $G$ by g.f. $(G)$.

Definition 3.1. A proper normal subgroup $H$ of a group $G$ is called a special generalized Frattini subgroup of $G$ provided that $G=N_{G}(A)$ for each normal subgroup $L$ of $G$ and each Hall subgroup $A$ of $L$ such that $G=H N_{G}(A)$. We denote the collection of all special generalized Frattini subgroups of $G$ by s.g.f. $(G)$.

Note that it follows immediately that if $H \in$ s.g.f. $(G)$, then $H \in$ g.f. $(G)$. If $H \in$ g.f. $(G)$, then it is not necessary for $H$ to be in s.g.f. $(G)$. In order to see this we give the following example which will also prove useful to us in other contexts.

ExAmple 3.2. (See 9.2.14 of [6] and Example 3.3 of [3]). Let $H=P \times K$ where $P$ is the Klein four-group and $K$ is cyclic of order 7. Let $G$ be the relative holomorph of $H$ by an automorphism of $H$ of order 3 which acts as an automorphism of order 3 on $P$ and on $K$. Then $|G|=84$, and $G=P K Q$ where $Q \in \operatorname{Syl}_{3}(G)$ is such that $N_{G}(Q)=Q$. Also $P$ and $K$ are normal in $G$. We note that both $P$ and $K$ are maximal generalized Frattini subgroups of $G$. Their product $P K$ is not a generalized Frattini subgroup of $G$. Since $K Q$ is a Hall subgroup of $G$ which is not normal in $G$, it follows that $P$ is not a special generalized Frattini subgroup of $G$. 
If $\theta$ is a homomorphism of $G$ onto $P Q$ with kernel $K$ such that $\theta$ restricted to $P$ is the identity on $P$, then $P \in$ g.f. $(G)$, but $P \theta \notin$ g.f. $(G \theta)$. This shows that a homomorphism does not necessarily preserve generalized Frattini subgroups even if its kernel is a generalized Frattini subgroup.

We note that if $G$ is a nilpotent group, then every proper normal subgroup of $G$ is a special generalized Frattini subgroup of $G$.

THEOREM 3.3. Let $H \in$ g.f. $(G)$, let $L$ be a normal subgroup of $G$, and $A$ be a nilpotent Hall $\pi$-subgroup of $L$ such that $G=H N_{G}(A)$. Then $A$ is a normal subgroup of $G$.

Proof. Let $p \in \pi$ and $P$ be the unique Sylow $p$-subgroup of $A$. Since $A$ is a Hall subgroup of $L$, it follows that $P \in \operatorname{Syl}_{p}(L)$. Since $N_{G}(A) \leqq N_{G}(P)$, we see that $G=H N_{G}(P)$. Thus $P$ is normal in $G$. Therefore, each Sylow subgroup of $A$ is normal in $G$, and so $A$ is normal in $G$.

Theorem 3.3 cannot be improved by requiring $A$ to be only supersolvable instead of nilpotent. To see this let $G$ be the group of order 84 of Example 3.2. Then $P$ is a generalized Frattini subgroup of $G$, and $K Q$ is a supersolvable Hall subgroup of $G$ such that $G=P N_{G}(K Q)$, but $G \neq N_{G}(K Q)$.

We now prove a few basic theorems about special generalized Frattini subgroups.

THEOREM 3.4. Let $H \in$ s.g.f. $(G)$. Then

(i) $H$ is nilpotent,

(ii) If $K$ is a normal subgroup of $G$ that is contained in $H$, then $K \in$ s.g.f. $(G)$,

(iii) $H \phi(G) \in$ s.g.f. $(G)$,

(iv) If $H Z(G)<G$, then $H Z(G) \in$ s.g.f. $(G)$.

Proof. (i) Let $P \in \operatorname{Syl}_{p}(H)$ where $p$ is a prime. Then $G=$ $H N_{G}(P)$, so $P$ is normal in $G$. Thus $H$ is nilpotent.

(ii) Let $K$ be a normal subgroup of $G$ contained in $H$. Let $L$ be a normal subgroup of $G$ and $A \in \operatorname{Hall}_{\pi}(L)$ such that $G=K N_{G}(A)$. Then $G=H N_{G}(A)$ which implies $G=N_{G}(A)$. Thus $K \in$ s.g.f. $(G)$.

(iii) $\dot{\phi}(G)$ is the set of nongenerators of $G$, hence $H \phi(G) \in$ s.g.f. $(G)$.

(iv) Since $Z(G) \leqq N_{G}(A)$ for any subgroup $A$ of $G$, (iv) follows easily.

Corollary 3.5. ( i ) $\phi(G) \in$ s.g.f. $(G)$.

(ii) If $G$ is nonabelian, then $Z(G) \in$ s.g.f. $(G)$. 
Recall that a group $G$ is said to be $\pi$-closed, $\pi$ is a set of primes, provided that the set of $\pi$-elements in $G$ forms a subgroup of $G$. The following properties are equivalent for the group $G$ : the group $G$ is $\pi$-closed; $G$ has a normal Hall $\pi$-subgroup; $G$ has a unique maximal $\pi$-subgroup. It is easy to show that subgroups and homomorphic images of $\pi$-closed groups are also $\pi$-closed.

If $H$ is a normal subgroup of $G$ such that $H$ is a $\pi$-group and $G / H$ is $\pi$-closed, then clearly $G$ is $\pi$-closed. If, however, $H$ and $G / H$ are $\pi$-closed, then it is not necessary for $G$ to be $\pi$-closed. Even if $H \in$ g.f. $(G)$ and $G / H$ is $\pi$-closed, $G$ may not be $\pi$-closed. For let $G$ be the group of order 84 of Example 3.2. If $\pi=\{3,7\}$ and $P \in \operatorname{Syl}_{2}(G)$, then $P \in$ g.f. $(G)$ and $G / P$ is a $\pi$-group. However, $G$ is not $\pi$-closed. We are thus led to make the following definition.

Definition 3.6. The proper normal subgroup $H$ of $G$ is said to satisfy property $\left(N_{\pi}\right), \pi$ is a set of primes, if and only if for any normal subgroup $K$ of $G$ containing $H$ such that $K / H$ is $\pi$-closed, then $K$ is $\pi$-closed.

Theorem 3.7. If $H \in$ s.g.f. $(G)$, then $H$ satisfies property $\left(N_{\pi}\right)$ for any set of primes $\pi$.

Proof. Let $H \in$ s.g.f. $(G)$, and let $K$ be a normal subgroup of $G$ that contains $H$ such that $K / H$ is $\pi$-closed, where $\pi$ is a set of primes. Then $K / H$ has a unique Hall $\pi$-subgroup $L / H$ which implies that $L$ is a normal subgroup of $G$. Since $H$ is nilpotent, it has a unique Hall $\pi^{\prime}$-subgroup $A$. Then $A$ is a normal subgroup of $G$ and $A \in \operatorname{Hall}_{\pi^{\prime}}(L)$ since $|L: A|=|L: H||H: A|$ is a $\pi$-number. Thus $A$ has a complement $B$ in $L$ by the Schur-Zessenhaus Theorem (see Theorem 9.3 of [6]).

We now show that $B$ is a normal Hall $\pi$-subgroup of $K$. Since $A \in \operatorname{Hall}_{\pi^{\prime}}(L)$, we see that $B \in \operatorname{Hall}_{\pi}(L)$. Now $A$ is solvable, so any two complements of $A$ in $L$ are conjugate in $L$ (see Theorem 9.3.9 of [6]). It follows that $G=L N_{G}(B)$. Therefore, $G=A B N_{G}(B)=$ $A N_{G}(B)=N_{G}(B)$, since $A \in$ s.g.f. $(G)$. Now $|K: B|=|K: L||L: B|$ is a $\pi^{\prime}$-number. Thus $B$ is a normal Hall $\pi$-subgroup of $K$ which implies that $K$ is $\pi$-closed. Therefore, $H$ satisfies property $\left(N_{\pi}\right)$ for each set of primes $\pi$. This completes the proof.

THEOREM 3.8. Let $H \in$ s.g.f. $(G)$ and $K$ be a subnormal subgroup of $G$ such that $K /(H \cap K)$ is $\pi$-closed. Then $K$ is $\pi$-closed.

Proof. $H K / H$ is a $\pi$-closed subnormal subgroup of $G / H$. Let $M / H$ be a maximal $\pi$-closed subnormal subgroup of $G / H$ which 
contains $H K / H$. Z. Janko proves in [5, p. 247] that a maximal $\pi$-closed subnormal subgroup of $G$ is normal in $G$. Thus $M$ is a normal subgroup of $G$ which is $\pi$-closed by Theorem 3.7. Since $K$ is a subgroup of $M, K$ is $\pi$-closed.

Definition 3.9. The proper normal subgroup $H$ of a group $G$ is said to satisfy property $\left(N_{\pi}^{\prime}\right), \pi$ is a set of primes, if and only if, for each subnormal subgroup $K$ of $G$ containing $H$ such that $K / H$ is $\pi$-closed, then $K$ is $\pi$-closed.

Definition 3.10. (See Baer, [1].) The normal subgroup $H$ of a finite group $G$ is a weakly hypercentral subgroup of $G$, if it has the following property:

(W) If $H$ is contained in the normal subgroup $K$ of $G$, if $x$ and $y$ are elements of $H$ and $K$ respectively, and if $|x|$ is relatively prime to $|y|$ and to $|K: H|$, then $x y=y x$.

THEOREM 3.11. The following statements are equivalent for the proper normal subgroup $H$ of the group $G$ :

(i) $H \in$ s.g.f. $(G)$.

(ii) $H$ satisfies property $\left(N_{\pi}^{\prime}\right)$ for any set of primes $\pi$.

(iii) $H$ satisfies property $\left(N_{\pi}\right)$ for any set of primes $\pi$.

(iv) $H$ is a weakly hypercentral subgroup of $G$.

Proof. (i) implies (ii) by Theorem 3.8. It is clear that (ii) implies (iii). We now show that (iii) implies (i). Let $L$ be a normal subgroup of $G$, and let $A \in \operatorname{Hall}_{\pi}(L), \pi$ is a set of primes, such that $G=H N_{G}(A)$. Then $H A$ is a normal subgroup of $G$ and $H A / H$ is a $\pi$-group. Since $H$ satisfies property $\left(N_{\pi}\right), H A$ is $\pi$-closed. Then $H A \cap L$ is $\pi$-closed and $A \in \operatorname{Hall}_{\pi}(H A \cap L)$. Therefore, $A$ is a normal subgroup of $G$ and so $H \in$ s.g.f. $(G)$. Thus (i), (ii), and (iii) are equivalent statements.

If $H$ is a weakly hypercentral subgroup of $G$, then $H$ satisfies property $\left(N_{\pi}\right)$ for each set of primes $\pi$ by Corollary 1 of [1, p. 637].

Now suppose that $H$ satisfies the first three equivalent statements. R. Baer has shown in [1, p. 636] that $H$ is weakly hypercentral in $G$ if and only if $H$ is nilpotent and if $H$ is contained in the normal subgroup $K$ of $G$, then the totality of $\pi$-elements in $K$ is a subgroup of $K$ where $\pi$ is the set of prime divisors of $|K: H|$. Since $H \in$ s.g.f. $(G)$, we have that $H$ is nilpotent. Now let $K$ be a normal subgroup of $G$ containing $H$ and let $\pi$ denote the set of primes dividing $|K: H|$. Then $K / H$ is a $\pi$-group and so $K$ is $\pi$-closed. Hence by Baer's characterization, we see that $H$ is a weakly hypercentral subgroup of $G$. This completes the proof. 
COROLlaRY 3.12. Let $H \in$ s.g.f. $(G)$ and let $K$ be a proper normal subgroup of $G$ which contains $H$. Then $K \in$ s.g.f. $(G)$ if and only if $K / H \in$ s.g.f. $(G / H)$.

Proof. Suppose that $K \in$ s.g.f. $(G)$, and $L / H$ is a normal subgroup of $G / H$ which contains $K / H$ such that $L / H / K / H$ is $\pi$-closed, where $\pi$ is a set of primes. Then $L$ is a normal subgroup of $G$ such that $L / K$ is $\pi$-closed. By Theorem 3.11, $L$ is $\pi$-closed and so $L / H$ is $\pi$-closed. Thus $K / H \in$ s.g.f. $(G / H)$ by Theorem 3.11 .

Conversely, suppose that $K / H \in$ s.g.f. $(G / H)$. Let $L$ be a normal subgroup of $G$ which contains $K$ such that $L / K$ is $\pi$-closed, where $\pi$ is a set of primes. Then $L / H / K / H$ is $\pi$-closed which implies that $L / H$ is $\pi$-closed. Thus $L$ is $\pi$-closed and so $K \in$ s.g.f. $(G)$ by Theorem 3.11. This coupletes the proof.

The previous corollary was proved in a different manner in [1, p. 638].

COROLLARY 3.13. If $G$ is a nonnilpotent group, then $L(G)$ and the hypercenter $Z^{*}(G)$ are special generalized Frattini subgroups of $G$.

Proof. It can be shown that $L(G) / \phi(G)=Z(G / \phi(G))$. Thus $L(G) / \dot{\phi}(G) \in$ s.g.f. $(G / \dot{\phi}(G))$ and $\dot{\phi}(G) \in$ s.g.f. $(G)$. Hence $L(G) \in$ s.g.f. $(G)$ by Corollary 3.12. Since $Z^{*}(G) \leqq L(G)$, it follows that $Z^{*}(G) \in$ s.g.f. $(G)$.

CoROLlary 3.14. Let $H \in$ s.g.f. $(G)$ and let $K$ be a subnormal subgroup of $G$ which properly contains $H$. Then $H \in$ s.g.f. $(K)$.

Proof. Let $L$ be a normal subgroup of $K$ which contains $H$ such that $L / H$ is $\pi$-closed, where $\pi$ is a set of primes. Then $L$ is a subnormal subgroup of $G$ such that $L / H$ is $\pi$-closed. Since $H$ satisfies property $\left(N_{\pi}^{\prime}\right)$ with respect to $G$, we see that $L$ is $\pi$-closed. Thus $H$ satisfies property $\left(N_{\pi}\right)$ with respect to $K$, and so $H \in$ s.g.f. $(K)$.

DEFinition 3.15. A group $G$ is said to be a $\pi$-dissolved group, $\pi$ is a set of prime, if and only if the set of all $\pi$-elements of $G$ is a solvable subgroup of $G$.

THEOREM 3.16. If $H \in$ s.g.f. $(G)$ and $K$ is a subnormal subgroup of $G$ containing $H$ such that $K / H$ is $\pi$-dissolved, then $K$. is $\pi$ dissolved.

Proof. Let $H \in$ s.g.f. $(G)$ and let $K$ be a subnormal subgroup of 
$G$ such that $K / H$ is $\pi$-dissolved. By Theorem $3.8, K$ is $\pi$-closed. Let $A$ be the subgroup consisting of all $\pi$-elements in $K$. Then $A \cap H$ and $A H / H$ are solvable; whence $A$ is solvable. Thus $K$ is $\pi$-dissolved, and the proof is complete.

Since $\phi(G) \in$ s.g.f. $(G)$, we get the following result due to R. Baer [2, p. 135].

Corollary 3.17. A group $G$ is $\pi$-dissolved if and only if $G / \phi(G)$ is $\pi$-dissolved.

We note that the results of this section enable us to prove in a different manner some of the results of $R$. Baer in [1]. For example, Baer proves that if $H$ is a weakly hypercentral subgroup of $G$, then $H \phi(G)$ is also weakly hypercentral (see p. 644 of [1]). This follows by our methods from Theorem 3.4 (iii) and Theorem 3.11. From this we see that $\phi(G) \leqq H$ for each maximal weakly hypercentral subgroup $H$ of $G$. Baer defines the weak hypercenter $H_{W}(G)$ to be the intersection of all maximal weakly hypercentral subgroups of $G$. Thus $\phi(G) \leqq H_{V W}(G)$ by the above remarks. A slightly stronger result is the following.

Theorem 3.18. If $G$ is a group, then $L(G) \leqq H_{W}(G)$.

Proof. If $G$ is nilpotent, then the result is clear. If $G$ is nonnilpotent, then $L(G) / \phi(G)=Z(G / \dot{\phi}(G)) \leqq K / \dot{\phi}(G)$ for any maximal weakly hypercentral subgroup $K$ of $G$. This follows from Theorem 3.4 and Corollary 3.12. Thus $L(G) \leqq K$ which implies $L(G) \leqq H_{V W}(G)$.

4. Products of weakly hypercentral subgroups. We recall that the product of two generalized Frattini subgroups may not be a generalized Frattini subgroup, even if their orders are relatively prime (see Example 3.2). If, however, we require one of the subgroups to be weakly hypercentral and the other to be generalized Frattini of relatively prime order, then the product is a generalized Frattini subgroup. We first prove the following lemma.

Lemma 4.1. Let $K$ be a weakly hypercentral subgroup of $G$, and let $H$ be a normal subgroup of $G$. If $|H|$ and $|K|$ are relatively prime, then $K H / H$ is a weakly hypercentral subgroup of $G / H$.

Proof. We show that $K H / H$ satisfies the definition of weakly hypercentral subgroup. Let $M / H$ be a normal subgroup of $G / H$ which contains $K H / H$. Let $k H \in K H / H$ and $m H \in M / H$ where $k \in K$ and $m \in M$, be such that their orders are relatively prime and $|k H|$ 
is relatively prime to $|M / H: K H / H|$. It suffices to show that $k H$ and $m H$ commute.

Now there exists $m_{1} \in M$ such that $\left|m_{1}\right|$ is divisible only by the primes that divide $|m H|$ and such that $m_{1} H=m H$. Hence $\left|m_{1}\right|$ and $|k|$ are relative prime. It also follows that $|k|$ is relatively prime to $|M: K|$. Since $K$ is a weakly hypercentral subgroup of $G$, we see that $m_{1} k=k m_{1}$. Therefore $m H$ and $k H$ commute and the proof is complete.

THEOREM 4.2. Let $K$ be a weakly hypercentral subgroup of $G$ and $H$ be a subgroup of $G$ such that $|H|$ and $|K|$ are relatively prime and $H K$ is a proper subgroup of $G$. If $H \in$ g.f. $(G)$, then $H K \in$ g.f. $(G)$. If $H$ is weakly hypercentral, then $H K$ is weakly hypercentral.

Proof. If $H \in$ g.f. $(G)$, then $H K / H$ is a weakly hypercentral subgroup of $G / H$ by Lemma 4.1. Thus $H K / H \in$ g.f. $(G / H)$. By Theorem 3.4 of [3], $H K \in$ g.f. $(G)$. If $H$ is weakly hypercentral, $H K$ is weakly hypercentral by Lemma 4.1 and Corollary 3.12.

ExAMPLE 4.3. (Baer [1, p. 640].) In the previous theorem, we cannot delete the requirement that $|H|$ and $|K|$ are relatively prime. Let $p$ be an odd prime and $J_{p}$ denote the cyclic group of order $p$. Let $T$ be the automorphism of $J_{p} \times J_{p}$ that sends each element into its inverse. Let $G$ be the relative holomorph of $J_{p} \times J_{p}$ by $\langle T\rangle$. Then every cyclic subgroup of $J_{p} \times J_{p}$ is a weakly hypercentral subgroup of $G$, but $J_{p} \times J_{p}$ is not generalized Frattini.

THEOREM 4.4. Let $K$ be a weakly hypercentral subgroup of $G$ and $H \in$ g.f. $(G)$. If either $H$ or $K$ is a Hall subgroup of $G$, and if $H K$ is proper in $G$, then $H K \in$ g.f. $(G)$.

Proof. Let $p_{1}, \cdots, p_{n}$ be the primes that divide $|H|$, and let $q_{1}, \cdots, q_{r}$ be the primes that divide $|K|$. If $|H|$ is relatively prime to $|K|$, then the result follows from Theorem 4.2. Hence we may suppose that $p_{1}=q_{1}, \cdots, p_{k}=q_{k}$ are the only primes that divide both $|H|$ and $|K|$. For each $i$ between 1 and $k$, let $P_{i}$ be the Sylow $p_{i}$-subgroup of $H$ and $Q_{i}$ be the Sylow $q_{i}$-subgroup of $K$.

Now suppose that $H$ is a Hall subgroup of $G$. Then $Q_{i} \leqq P_{i}$ for $i=1, \cdots, k$. Now let $Q_{j}$ be the Sylow $q_{j}$-subgroup of $K$ for $j=k+1, \cdots, r$. Since $K$ is nilpotent, $Q_{k+1} \cdots Q_{r}$ is normal in $G$ and is thus a weakly hypercentral subgroup of $G$. Hence $H K=$ $H\left(Q_{k+1} \cdots Q_{r}\right)$ is a generalized Frattini subgroup of $G$ by Theorem 4.2.

If $K$ is a Hall subgroup of $G$, then we can prove in a similar 
manner that $H K \in$ g.f. $(G)$. This completes the proof.

We note that if $H$ and $K$ are weakly hypercentral subgroups of $G$, one of which is a Hall subgroup of $G$, then it can be shown that $H K$ is a weakly hypercentral subgroup of $G$. The proof is analogous to the proof of Theorem 4.4.

If the group $G$ possesses a unique minimal normal subgroup $M$, and if $G / M$ possesses a normal subgroup, not 1 , whose order is prime to the order of $M$, then R. Baer [2, p. 118] proved that $\phi(G)=1$. We now show that in such a group, the only weakly hypercentral subgroup is the identity.

THEOREM 4.5. If the group $G$ possesses a unique minimal normal subgroup $M$ and if $G / M$ possesses a normal subgroup, not 1 , whose order is prime to the order of $M$, then the only weakly hypercentral subgroup of $G$ is the identity subgroup. In particular, $\phi(G)=1$.

Proof. Suppose that $H$ is a nontrivial weakly hypercentral subgroup of $G$. Since $M$ is the unique minimal normal subgroup of $G$, we see that $M \leqq H$. Let $K / M$ be a nontrivial normal subgroup of $G / M$ whose order is prime to the order of $M$. Then $M$ is a normal Hall subgroup of $K$, and $M$ is solvable. Thus $M$ has a complement $S$ in $K$ by the Schur-Zassenhaus theorem (see Theorem 9.3.6 of [6]). Also any two complements of $M$ in $K$ are conjugate in $K$. It follows that $G=M N_{G}(S)$. Since $M \leqq H$, we see that $M \in$ s.g.f. $(G)$, and so $G=N_{G}(S)$. Thus $K=M \times S$ and $M \leqq S$, whence $M=1$ which is impossible. This completes the proof.

It is clear that by replacing $\pi$ by $\{p\}$ in the theorems of $\S$ 's 3 and 4, we get corresponding theorems for generalized Frattini subgroups. For example, N. Ito in [4] proves that a subnormal subgroup $K$ of $G$ is nilpotent if and only if its commutator subgroup $K^{\prime}$ is contained in $\phi(G)$. By Theorem 3.8, one can show that a subnormal subgroup $K$ of $G$ is nilpotent provided that $K^{\prime} \leqq H$ for some $H \in$ g.f. $(G)$. Also one can easily see that a subgroup $H$ is a generalized Frattini subgroup of $G$ if and only if $H$ is a normal nilpotent subgroup of $G$ with the property that $F(G / H)=F(G) / H$, where $F(G)$ denotes the Fitting subgroup of $G$.

\section{REFERENCES}

1. R. Baer, Nilpotent characteristic subgroups of finite groups, Amer. J. Math. 75 (1953), 633-664.

2. Classes of finite groups and their properties, Illinois J. Math. 1 (1957), 
115-187.

3. J. C. Beidleman and T. K. Seo, Generalized Frattini subgroups of finite groups, Pacific J. Math. 23 (1967), 441-450.

4. N. Ito, Uber die Frattini-Gruppe einer endlichen Gruppe, Proc. Japan Acad. 31 (1955), 327-328.

5. Z. Janko, Eine Bemerkung uber die $\phi$-Untergruppe endlicher Gruppen, Acta Sci Math. (Szeged) 23 (1962), 247-248.

6. W. R. Scott, Group theory, Prentice Hall, New Jersey, 1964.

Received September 16, 1968. The results presented in this paper form a portion of the author's Ph. D. dissertation written under the direction of Dr. J. C. Beidleman. The author was supported by a University of Kentucky Doctoral Year Fellowship.

Kent State University

KENT, OHIO 


\section{PACIFIC JOURNAL OF MATHEMATICS}

\section{EDITORS}

\author{
H. ROYDEN \\ Stanford University \\ Stanford, California \\ Richard Pierce \\ University of Washington \\ Seattle, Washington 98105
}

\author{
J. DugundJI \\ Department of Mathematics \\ University of Southern California \\ Los Angeles, California 90007 \\ BASIL GoRDON \\ University of California \\ Los Angeles, California 90024
}

\section{ASSOCIATE EDITORS}
E. F. BECKENBACH
B. H. Neumann
F. WOLF
K. YoSHIDA

\section{SUPPORTING INSTITUTIONS}

\author{
UNIVERSITY OF BRITISH COLUMBIA \\ CALIFORNIA INSTITUTE OF TECHNOLOGY \\ UNIVERSITY OF CALIFORNIA \\ MONTANA STATE UNIVERSITY \\ UNIVERSITY OF NEVADA \\ NEW MEXICO STATE UNIVERSITY \\ OREGON STATE UNIVERSITY \\ UNIVERSITY OF OREGON \\ OSAKA UNIVERSITY \\ UNIVERSITY OF SOUTHERN CALIFORNIA
}

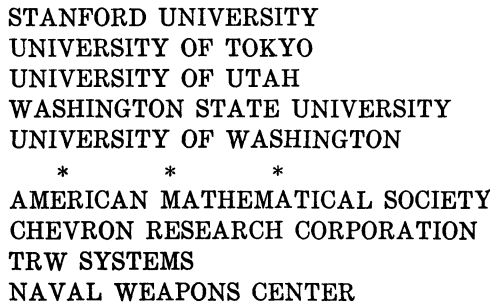

The Supporting Institutions listed above contribute to the cost of publication of this Journal, but they are not owners or publishers and have no responsibility for its content or policies.

Mathematical papers intended for publication in the Pacific Journal of Mathematics should be in typed form or offset-reproduced, double spaced with large margins. Underline Greek letters in red, German in green, and script in blue. The first paragraph or two must be capable of being used separately as a synopsis of the entire paper. It should not contain references to the bibliography. Manuscripts, in duplicate if possible, may be sent to any one of the four editors. Please classify according to the scheme of Math. Rev. 36, 1539-1546. All other communications to the editors should be addressed to the managing editor, Richard Arens, University of California, Los Angeles, California, 90024.

50 reprints are provided free for each article; additional copies may be obtained at cost in multiples of 50 .

The Pacific Journal of Mathematics is published monthly. Effective with Volume 16 the price per volume (3 numbers) is $\$ 8.00$; single issues, $\$ 3.00$. Special price for current issues to individual faculty members of supporting institutions and to individual members of the American Mathematical Society: $\$ 4.00$ per volume; single issues $\$ 1.50$. Back numbers are available.

Subscriptions, orders for back numbers, and changes of address should be sent to Pacific Journal of Mathematics, 103 Highland Boulevard, Berkeley, California, 94708.

PUBLISHED BY PACIFIC JOURNAL OF MATHEMATICS, A NON-PROFIT CORPORATION

Printed at Kokusai Bunken Insatsusha (International Academic Printing Co., Ltd.), 7-17, Fujimi 2-chome, Chiyoda-ku, Tokyo, Japan. 


\section{Pacific Journal of Mathematics}

\section{Vol. 31, No. $2 \quad$ December, 1969}

Efraim Pacillas Armendariz, Quasi-injective modules and stable torsion

classes..........................................

J. Adrian (John) Bondy, On Ulam's conjecture for separable graphs...

Vasily Cateforis and Francis Louis Sandomierski, On commutative rings over which the singular submodule is a direct summand for every module .....

Rafael Van Severen Chacon, Approximation of transformations with continuous

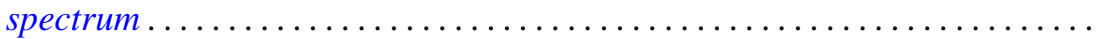

Raymond Frank Dickman and Alan Zame, Functionally compact spaces ...... 303

Ronald George Douglas and Walter Rudin, Approximation by inner

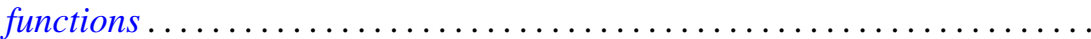

John Walter Duke, A note on the similarity of matrix and its conjugate

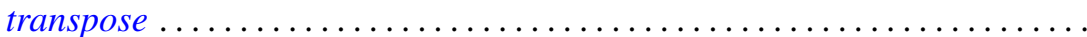

Micheal Neal Dyer and Allan John Sieradski, Coverings of mapping

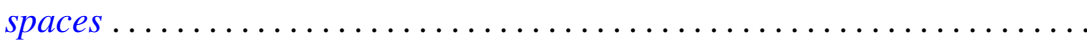

Donald Campbell Dykes, Weakly hypercentral subgroups of finite groups .....

Nancy Dykes, Mappings and realcompact spaces.....................

Edmund H. Feller and Richard Laham Gantos, Completely injective

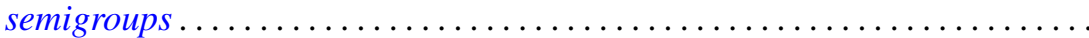

Irving Leonard Glicksberg, Semi-square-summable Fourier-Stieltjes

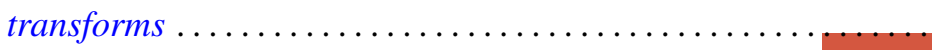

Samuel Irving Goldberg and Kentaro Yano, Integrability of almost cosymplectic structures...

Seymour Haber and Charles Freeman Osgood, On the sum $\sum\langle n \alpha\rangle^{-t}$ and numerical integration ..........................

Sav Roman Harasymiv, Dilations of rapidly decreasing functions ....

William Leonard Harkness and R. Shantaram, Convergence of a sequence of

transformations of distribution functions

Herbert Frederick Kreimer, Jr., A note on the outer Galois theory of rings ...

James Donald Kuelbs, Abstract Wiener spaces and applications to analysis. .

Roland Edwin Larson, Minimal $T_{0}$-spaces and minimal $T_{D}$-spaces...

A. Meir and Ambikeshwar Sharma, On Ilyeff's conjecture .

Isaac Namioka and Robert Ralph Phelps, Tensor products of compact convex sets....

James L. Rovnyak, On the theory of unbounded Toeplitz operators ....

Benjamin L. Schwartz, Infinite self-interchange graphs.......

George Szeto, On the Brauer splitting theorem...

Takayuki Tamura, Semigroups satisfying identity $x y=f(x$,

Kenneth Tolo, Factorizable semigroups .................. 